Supplement of The Cryosphere Discuss., 8, 4075-4126, 2014

http://www.the-cryosphere-discuss.net/8/4075/2014/

doi:10.5194/tcd-8-4075-2014-supplement

(C) Author(s) 2014. CC Attribution 3.0 License.

(c) (1)

Supplement of

\title{
Post-LIA glacier changes along a latitudinal transect in the Central Italian Alps
}

R. Scotti et al.

Correspondence to: F. Brardinoni (francesco.brardinoni@unimib.it) 
1 Post-LIA glacier changes along a climatic transect in the Central

2 Italian Alps: Supplement Material

3

4 Supplementary Table S1. Glacier characteristics in the three sub-regions in year 2007.

\begin{tabular}{|c|c|c|c|c|}
\hline \multicolumn{2}{|c|}{ Attribute } & \multicolumn{3}{|c|}{ Sub-region } \\
\hline & & Livigno & Disgrazia & Orobie \\
\hline \multirow{3}{*}{$\mathrm{GS}\left(\mathrm{km}^{2}\right)$} & Mean & 0.07 & 0.20 & 0.04 \\
\hline & Median & 0.02 & 0.04 & 0.02 \\
\hline & Range & $0.003-0.37$ & $0.002-2.31$ & $0.002-0.22$ \\
\hline \multirow{3}{*}{$\mathrm{ABR}$} & Low & - & 3 & - \\
\hline & Moderate & 4 & 5 & - \\
\hline & High & 12 & 29 & 44 \\
\hline \multirow{3}{*}{$\mathrm{E}_{\min }(\mathrm{m}$ a.s.l.) } & Mean & 2803 & 2788 & 2425 \\
\hline & Median & 2765 & 2795 & 2451 \\
\hline & Range & $(2707-3032)$ & $(2229-3191)$ & $(2030-2852)$ \\
\hline \multirow{3}{*}{$\mathrm{ELA}_{0}$ (m a.s.1.) } & Mean & 2864 & 2887 & 2473 \\
\hline & Median & 2833 & 2890 & 2517 \\
\hline & Range & (2772-3047) & $(2440-3210)$ & $(2047-2879)$ \\
\hline \multirow{3}{*}{$\mathrm{E}_{\max }$ (m a.s.l.) } & Mean & 2973 & 3065 & 2604 \\
\hline & Median & 2989 & 3083 & 2584 \\
\hline & Range & $(2795-3178)$ & (2476-3634) & $(2123-2796)$ \\
\hline $\mathrm{E}_{\mathrm{rc}}(\mathrm{m}$ a.s.l. $)$ & Median & 2974 & 3109 & 2730 \\
\hline \multirow{3}{*}{$S\left({ }^{\circ}\right)$} & Mean & 27.8 & 28.7 & 30.0 \\
\hline & Median & 29.2 & 27.1 & 29.1 \\
\hline & Range & $(19.6-33.0)$ & $(18.1-45.0)$ & $(18.8-42.2)$ \\
\hline \multirow{3}{*}{$\operatorname{CSR}\left(\mathrm{W} \mathrm{m}^{2}\right)$} & Mean & 176 & 210 & 149 \\
\hline & Median & 172 & 213 & 145 \\
\hline & Range & $(152-218)$ & $(121-258)$ & $(94-221)$ \\
\hline \multirow{3}{*}{$\operatorname{MAP}\left(\mathrm{mm} \mathrm{a}^{-1}\right)$} & Mean & 1070 & 1295 & 1680 \\
\hline & Median & 1065 & 1301 & 1682 \\
\hline & Range & $(790-1200)$ & $(1210-1370)$ & $(1620-1770)$ \\
\hline
\end{tabular}

5

6 
1 Supplementary Table S2. Glacier count and area from 1860 to 2007 stratified in size classes.

\begin{tabular}{lcccccccccc}
\hline \multicolumn{10}{c}{ Livigno } \\
\hline Size Classes & \multicolumn{1}{c}{$\mathbf{1 8 6 0}$} & \multicolumn{2}{c}{$\mathbf{1 9 5 4}$} & \multicolumn{1}{c}{$\mathbf{1 9 9 0}$} & \multicolumn{2}{c}{$\mathbf{2 0 0 3}$} & \multicolumn{2}{c}{$\mathbf{2 0 0 7}$} \\
$\mathrm{Km}^{2}$ & Count & Area & Count & Area & Count & Area & Count & Area & Count & Area \\
\hline & & $\mathrm{Km}^{2}$ & & $\mathrm{Km}^{2}$ & & $\mathrm{Km}^{2}$ & & $\mathrm{Km}^{2}$ & & $\mathrm{Km}^{2}$ \\
$<0.1$ & 5 & 0.2 & 13 & 0.4 & 16 & 0.5 & 18 & 0.4 & 13 & 0.3 \\
$0.1-0.5$ & 6 & 1.3 & 6 & 1.1 & 5 & 1.2 & 3 & 0.9 & 3 & 0.8 \\
$0.5-1$ & 2 & 1.4 & 2 & 1.0 & 1 & 0.6 & - & - & - & - \\
$1.0-2.0$ & 2 & 2.5 & - & - & - & - & - & - & - & - \\
Total & 15 & 5.4 & 21 & 2.5 & 22 & 2.3 & 21 & 1.3 & 16 & 1.1 \\
& & \pm 0.53 & & \pm 0.20 & & \pm 0.07 & & \pm 0.03 & & \pm 0.02 \\
\hline
\end{tabular}

\section{Disgrazia}

\begin{tabular}{lcccccccccc}
$<0.1$ & 6 & 0.4 & 17 & 0.8 & 21 & 0.9 & 28 & 1.0 & 26 & 0.6 \\
$0.1-0.5$ & 12 & 3.0 & 13 & 2.8 & 12 & 2.7 & 7 & 1.6 & 7 & 1.4 \\
$0.5-1$ & 3 & 2.2 & 3 & 2.4 & 2 & 1.5 & 2 & 1.3 & 2 & 1.1 \\
$1.0-2.0$ & 3 & 3.7 & 1 & 1.1 & 1 & 1.1 & - & - & 1 & 1.9 \\
$2.0-5.0$ & 2 & 5.6 & 2 & 5.3 & 2 & 5.7 & 2 & 4.6 & 1 & 2.3 \\
$5.0-10.0$ & 1 & 7.0 & - & - & - & - & - & - & - & - \\
Total & 27 & 22.0 & 36 & 12.4 & 38 & 11.9 & 39 & 8.4 & 37 & 7.3 \\
& & \pm 1.28 & & \pm 0.59 & & \pm 0.22 & & \pm 0.10 & & \pm 0.09 \\
\hline
\end{tabular}

\section{Orobie}

\begin{tabular}{lcccccccccc}
$<0.1$ & 25 & 1.1 & 41 & 1.4 & 41 & 1.4 & 42 & 1.0 & 39 & 0.9 \\
$0.1-0.5$ & 18 & 3.9 & 8 & 1.8 & 8 & 1.7 & 6 & 1.0 & 5 & 0.9 \\
$0.5-1$ & 1 & 0.6 & - & - & - & - & - & - & - & - \\
$1.0-2.0$ & 1 & 1.1 & - & - & - & - & - & - & - & - \\
Total & 45 & 6.7 & 49 & 3.2 & 49 & 3.1 & 48 & 2.0 & 44 & 1.8 \\
& & \pm 0.93 & & \pm 0.31 & & \pm 0.12 & & \pm 0.06 & & \pm 0.05 \\
\hline
\end{tabular}

3 Supplementary Table S3. Correlation matrix for 10 variables in Livigno sub-region. Correlation coefficients $4 \geq 0.4$ are typed in bold.

\begin{tabular}{|c|c|c|c|c|c|c|c|c|c|c|}
\hline Livigno & $\mathrm{AC}(\%)$ & GS & MA & S & $\mathrm{E}_{\min }$ & $\mathrm{E}_{\max }$ & $\Delta \mathrm{E}$ & MAP & $\mathrm{E}_{\mathrm{rc}}$ & CSR \\
\hline $\mathrm{AC}(\%)$ & 1.00 & & & & & & & & & \\
\hline GS & 0.43 & 1.00 & & & & & & & & \\
\hline MA & -0.22 & -0.31 & 1.00 & & & & & & & \\
\hline S & -0.38 & -0.34 & 0.33 & 1.00 & & & & & & \\
\hline $\mathrm{E}_{\min }$ & -0.46 & -0.74 & 0.37 & 0.42 & 1.00 & & & & & \\
\hline $\mathrm{E}_{\max }$ & 0.72 & 0.67 & 0.02 & -0.53 & -0.54 & 1.00 & & & & \\
\hline$\Delta \mathrm{E}$ & 0.65 & 0.81 & -0.23 & -0.55 & -0.91 & 0.84 & 1.00 & & & \\
\hline MAP & -0.20 & -0.03 & 0.05 & -0.24 & 0.20 & -0.19 & -0.23 & 1.00 & & \\
\hline $\mathrm{E}_{\mathrm{rc}}$ & 0.77 & 0.65 & 0.12 & -0.37 & -0.44 & 0.87 & 0.70 & -0.02 & 1.00 & \\
\hline CSR & 0.43 & 0.36 & -0.09 & -0.88 & -0.26 & 0.77 & 0.57 & 0.23 & 0.56 & 1.00 \\
\hline
\end{tabular}


1 Supplementary Table S4. Correlation matrix for 10 variables in Disgrazia sub-region. Correlation

2 coefficients $\geq 0.4$ are typed in bold.

\begin{tabular}{lcccccccccc}
\hline Disgrazia & AC $(\boldsymbol{\%})$ & $\mathrm{GS}$ & $\mathrm{MA}$ & $\mathrm{S}$ & $\mathrm{E}_{\min }$ & $\mathrm{E}_{\max }$ & $\Delta \mathrm{E}$ & $\mathrm{MAP}$ & $\mathrm{E}_{\mathrm{rc}}$ & $\mathrm{CSR}$ \\
\hline $\mathbf{A C}(\boldsymbol{\%})$ & 1.00 & & & & & & & & & \\
$\mathrm{GS}$ & $\mathbf{0 . 4 2}$ & 1.00 & & & & & & & & \\
$\mathrm{MA}$ & 0.01 & -0.13 & 1.00 & & & & & & & \\
$\mathrm{~S}$ & -0.14 & -0.12 & -0.08 & 1.00 & & & & & & \\
$\mathrm{E}_{\min }$ & -0.28 & $\mathbf{- 0 . 7 4}$ & $\mathbf{0 . 5 3}$ & 0.17 & 1.00 & & & & & \\
$\mathrm{E}_{\max }$ & $\mathbf{0 . 4 5}$ & $\mathbf{0 . 6 3}$ & $\mathbf{0 . 5 1}$ & 0.00 & -0.18 & 1.00 & & & & \\
$\Delta \mathrm{E}$ & $\mathbf{0 . 4 7}$ & $\mathbf{0 . 8 9}$ & -0.06 & -0.12 & $\mathbf{- 0 . 8 1}$ & $\mathbf{0 . 7 3}$ & 1.00 & & & \\
$\mathrm{MAP}$ & -0.13 & 0.12 & 0.21 & $\mathbf{0 . 5 0}$ & 0.07 & 0.30 & 0.13 & 1.00 & & \\
$\mathrm{E}_{\mathrm{rc}}$ & 0.35 & 0.19 & $\mathbf{0 . 7 3}$ & 0.15 & 0.26 & $\mathbf{0 . 8 5}$ & 0.33 & 0.29 & 1.00 & \\
$\mathrm{CSR}$ & 0.11 & -0.07 & $\mathbf{0 . 8 4}$ & -0.32 & $\mathbf{0 . 4 1}$ & $\mathbf{0 . 5 2}$ & 0.00 & 0.05 & $\mathbf{0 . 7 1}$ & 1.00 \\
\hline
\end{tabular}

4 Supplementary Table S5. Correlation matrix in Orobie sub-region. Correlation coefficients $\geq 0.4$ are marked 5 in bold type.

\begin{tabular}{lcccccccccc}
\hline Orobie & AC $(\boldsymbol{\%})$ & $\mathrm{GS}$ & $\mathrm{MA}$ & $\mathrm{S}$ & $\mathrm{E}_{\min }$ & $\mathrm{E}_{\max }$ & $\Delta \mathrm{E}$ & $\mathrm{MAP}$ & $\mathrm{E}_{\mathrm{rc}}$ & $\mathrm{CSR}$ \\
\hline $\mathbf{A C}(\boldsymbol{\%})$ & 1.00 & & & & & & & & & \\
$\mathrm{GS}$ & -0.06 & 1.00 & & & & & & & & \\
$\mathrm{MA}$ & -0.15 & -0.04 & 1.00 & & & & & & & \\
$\mathrm{~S}$ & 0.00 & -0.26 & -0.13 & 1.00 & & & & & & \\
$\mathrm{E}_{\min }$ & $\mathbf{- 0 . 4 0}$ & -0.32 & 0.30 & 0.05 & 1.00 & & & & & \\
$\mathrm{E}_{\max }$ & -0.20 & $\mathbf{0 . 4 8}$ & 0.03 & 0.03 & 0.24 & 1.00 & & & & \\
$\Delta \mathrm{E}$ & 0.20 & $\mathbf{0 . 6 4}$ & -0.24 & -0.02 & $\mathbf{- 0 . 6 8}$ & $\mathbf{0 . 5 4}$ & 1.00 & & & \\
MAP & 0.25 & -0.03 & 0.33 & 0.23 & -0.05 & -0.12 & -0.05 & 1.00 & & \\
$\mathrm{E}_{\mathrm{rc}}$ & -0.03 & 0.31 & -0.06 & 0.01 & 0.19 & $\mathbf{0 . 7 5}$ & $\mathbf{0 . 4 0}$ & -0.01 & 1.00 & \\
CSR & -0.35 & 0.29 & 0.58 & $\mathbf{- 0 . 5 1}$ & 0.34 & 0.21 & -0.12 & -0.09 & 0.08 & 1.00 \\
\hline
\end{tabular}

6

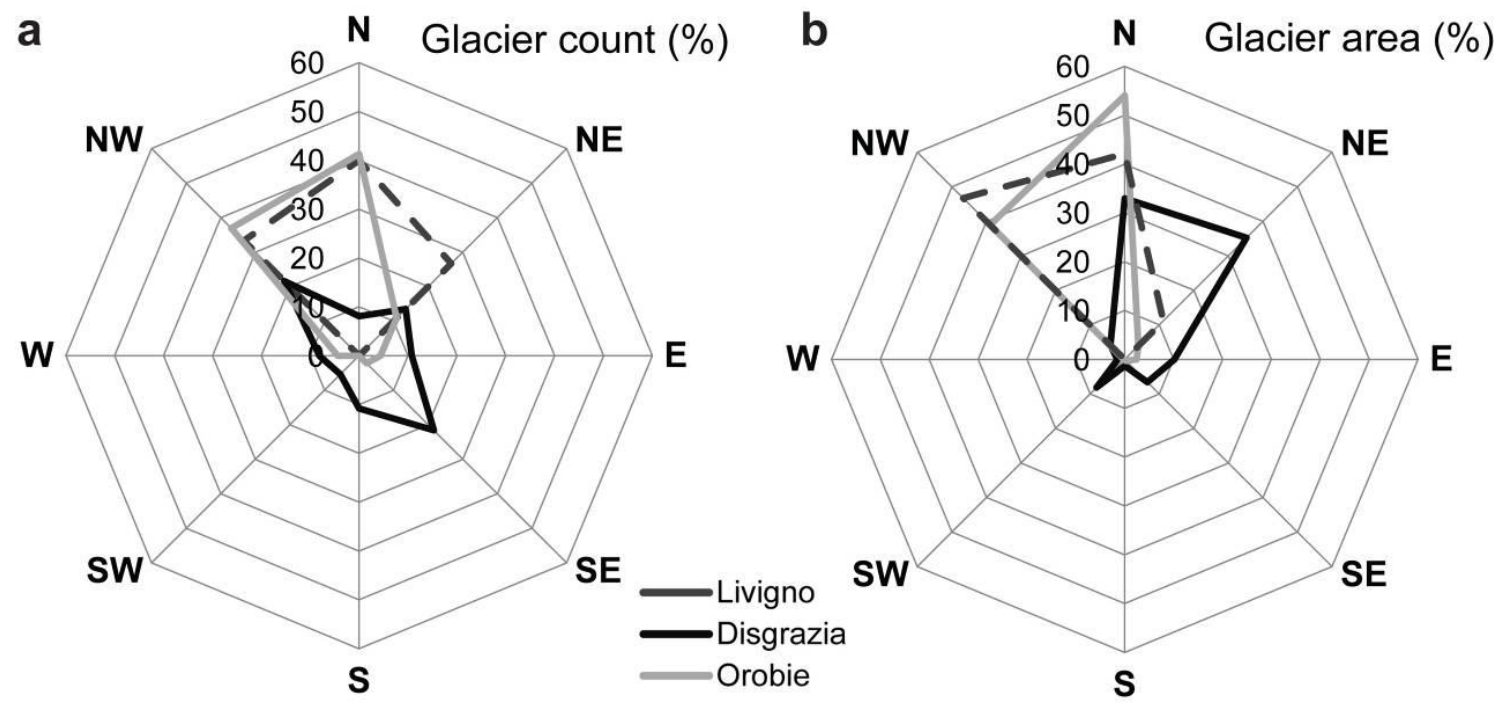

Supplementary Figure S1. Spider-web charts detailing the relative glacier abundance in number (a) and area 9 (b) across slope aspects in the three sub-regions. 